\title{
Associations Between the Kynurenine Pathway, Proinflammatory Cytokines, and Brain-Derived Neurotrophic Factor in Hospitalized Patients With Chronic Schizophrenia: A Preliminary Study
}

\author{
Naomichi Okamoto, Tomoya Natsuyama, Ryohei Igata, Yuki Konishi, Hirofumi Tesen, \\ Atsuko Ikenouchi and Reiji Yoshimura*
}

Department of Psychiatry, University of Occupational and Environmental Health Japan, Kitakyushu, Japan

\section{OPEN ACCESS}

Edited by:

Jolanta Kucharska-Mazur,

Pomeranian Medical

University, Poland

Reviewed by:

Laiana Quagliato,

Federal University of Rio de

Janeiro, Brazi

Gayathri Sundaram,

St Vincent's Hospital Sydney, Australia

${ }^{*}$ Correspondence:

Reiji Yoshimura

yoshi621@med.uoeh-u.ac.jp

Specialty section:

This article was submitted to

Psychopathology,

a section of the journal

Frontiers in Psychiatry

Received: 16 April 2021 Accepted: 28 June 2021

Published: 28 July 2021

Citation:

Okamoto N, Natsuyama T, Igata $R$, Konishi Y, Tesen H, Ikenouchi $A$ and Yoshimura R (2021) Associations Between the Kynurenine Pathway,

Proinflammatory Cytokines, and Brain-Derived Neurotrophic Factor in Hospitalized Patients With Chronic Schizophrenia: A Preliminary Study.

Front. Psychiatry 12:696059.

doi: 10.3389/fpsyt.2021.696059
Purpose: The kynurenine (Kyn) pathway may play a role in the pathophysiology of schizophrenia. This pathway shows crosstalk with proinflammatory cytokines, including interleukin-1 $\beta$ (IL-1 $\beta)$, IL-6, and tumor necrosis factor- $\alpha$ (TNF- $\alpha)$, and/or brain-derived neurotrophic factor (BDNF). Moreover, Kyn metabolites affect neurotransmission and cause neurotoxicity. To date, the influence of the Kyn pathway on proinflammatory cytokines and BDNF remains to be fully elucidated. The aim of this study was to investigate the relationships of the Kyn pathway with proinflammatory cytokines, BDNF, and psychiatric symptoms in patients with schizophrenia.

Methods: Thirty patients with schizophrenia and ten healthy control participants were recruited for this study. All patients were diagnosed with schizophrenia using the Diagnostic and Statistical Manual for Mental Disorders, Fifth Edition (DSM-5). The healthy controls were those who did not fulfill any of the diagnostic criteria in the DSM-5. The serum levels of Kyn and its metabolites, proinflammatory cytokines, and BDNF were measured in patients with schizophrenia and healthy controls. Patients with schizophrenia were also assessed for psychiatric symptoms using the Positive and Negative Syndrome Scale (PANSS).

Results: Patients with schizophrenia and healthy controls showed no significant differences in the levels of Kyn and its metabolites, proinflammatory cytokines, and BDNF. A significant positive correlation was found between the serum levels of TNF- $\alpha$ and Kyn $(r=0.53, p=0.0026)$ and the Kyn/tryptophan (Trp) value $(r=0.67, p=0.000046)$ in the schizophrenia group, but not in the healthy control group.

Conclusion: TNF- $\alpha$ affects the Kyn pathway in patients with chronic schizophrenia, but not in the healthy individuals, although serum TNF- $\alpha$ levels showed no difference between the two groups. Associations between the Kyn pathway and the levels of proinflammatory cytokines and BDNF or psychotic symptoms might be complicated in hospitalized patients with chronic schizophrenia.

Keywords: schizophrenia, kynurenine pathway, kynurenine, quinolinic acid, inflammatory cytokines, BDNF 


\section{INTRODUCTION}

Although the pathophysiology of schizophrenia is currently unclear, inflammation has been suggested to play an important role in the pathophysiology $(1,2)$. In addition, there is growing evidence of an interaction between inflammation and the kynurenine (Kyn) pathway in schizophrenia (3). The Kyn pathway is regulated by the immune system, and the decomposition of tryptophan via the Kyn pathway is activated by proinflammatory cytokines. The Kyn pathway is also considered to crosstalk with the immune system, proinflammatory cytokines, and neurotrophic factors $(4,5)$. Tryptophan (Trp) is degraded to Kyn, which is catabolized to either kynurenic acid (KynA) via kynurenine aminotransferases or to 3hydroxykynurenine (3-HK) via kynurenine 3-monooxygenase and finally to quinolinic acid (QA). Proinflammatory cytokines, including interleukin-1 $\beta$ (IL-1 $\beta$ ), IL-6, and tumor necrosis factor- $\alpha$ (TNF- $\alpha$ ), are thought to contribute to the pathogenesis of psychiatric symptoms in schizophrenia by Kyn pathway activation.

Kyn metabolites affect neurotransmission and cause neurotoxicity. Several studies have indicated that proinflammatory cytokines and the Kyn pathway in the blood are dysregulated in patients with schizophrenia. Increase in the plasma levels of proinflammatory cytokines, such as IL-1 $\beta$, IL- 6 , and TNF- $\alpha$, have been consistently reported in patients with schizophrenia (6). C-reactive protein (CRP) levels have also been reported to be elevated in patients with schizophrenia (7). Thus, these proinflammatory cytokines may accelerate the Kyn pathway (8), which may be related to changes in neurotrophic factors, which are associated with the symptoms of schizophrenia. According to the review (9), the Kyn pathway of Trp degradation generates several neuroactive compounds. KynA is an N-methyl-d-aspartate (NMDA) and alpha 7 nicotinic receptor antagonist. The KynA hypothesis of schizophrenia is based on the fact that KynA blocks glutamate receptors and is elevated in schizophrenia. KynA regulates glutamatergic and dopaminergic neurotransmission and elevated brain levels appear associated with psychotic symptoms and cognitive impairments in schizophrenia. Contributing to enhanced production of $\mathrm{KynA}$, the expression and enzyme activity of kynurenine 3-monooxygenase (KMO) are reduced in schizophrenia. There were several works by Engberg and Erhardt. The authors reported cerebrospinal fluid (CSF) levels of Kyn, and KynA in schizophrenia patients $(10,11)$. They also reported CSF levels of Kyn and KynA were elevated in patients with chronic schizophrenia, indicating the idea of brain immune activation in patients with schizophrenia, which suggested that IL-6 induces the Kyn pathway, leading to increased production of the NMDA receptor antagonist KynA in patients with schizophrenia (12). A recent report demonstrated that proinflammatory cytokines are involved in dorsolateral prefrontal cortex volume loss and attention impairment via the Kyn pathway (13). However, the associations among these components are complicated and controversial. Recent review reported the following findings, (1) schizophrenia patients with prescribed antipsychotic drugs had significantly higher Kyn levels compared with controls; (2) higher Kyn levels in CSF, lower plasma Kyn levels compared with controls; (3) the Kyn levels were higher in schizophrenia patients after treatment with antipsychotic drugs compared with baseline (14). Therefore, the correlations among proinflammatory cytokines, neurotrophic factors, and the Kyn pathway and their possible relevance to schizophrenic pathophysiology, must be further elucidated.

Thus, the aim of the present study was to investigate the associations of proinflammatory cytokines, including IL-1 $\beta$, IL6 , TNF- $\alpha$, and IL-10, a suppressive cytokine, and brain-derived neurotrophic factor (BDNF) with the Kyn pathway, which might be related to the symptomatology of schizophrenia patients. The present study aimed to shed light on how coordination among the Kyn pathway, proinflammatory cytokines, and BDNF may influence the symptomatology in chronic hospitalized schizophrenia patients.

\section{METHODS}

\section{Participants and Ethics Statement}

Thirty patients with schizophrenia participated in this study. The patients were recruited from the Komine-Eto Hospital and Shinmoji Hospital. All patients were diagnosed with schizophrenia using the Diagnostic and Statistical Manual for Mental Disorders, Fifth Edition (DSM-5) (15). Exclusion criteria included a history of major neurological disease, uncontrolled major medical illness, epilepsy, cerebrovascular accident, head trauma with cognitive sequelae, and intellectual disability. All patients with schizophrenia were taking antipsychotic medications. The control group of healthy controls did not currently have a DSM-5 applicable psychiatric diagnosis, nor did they have a family history of psychosis. All participants provided verbal and written informed consent. The research protocol was approved by the Ethics Committee of the University of Occupational and Environmental Health (Approval Number: UOEHCRB19-024).

\section{Clinical Assessment and Blood Sampling}

Patients with schizophrenia were assessed for clinical and neuropsychiatric symptoms using the Positive and Negative Syndrome Scale (PANSS) (16) and the Drug Induced ExtraPyramidal Symptoms Scale (DIEPSS) (17). Blood samples were collected between 7 and 9 a.m. before breakfast. Participants fasted and rested for at least $30 \mathrm{~min}$ prior to blood collection.

\section{Measurement of Metabolites of the Kyn Pathway}

The samples obtained at the University of Occupational and Environmental Health were transferred to Human Metabolome Technologies Inc. (HMT; Tsuruoka, Japan), where each 50- $\mu \mathrm{L}$ sample was mixed with $450 \mu \mathrm{L}$ of methanol containing internal standards $(10 \mu \mathrm{M})$ and vortexed. Chloroform $(500 \mu \mathrm{L})$ and Milli-Q water $(200 \mu \mathrm{L})$ were added, mixed thoroughly, and centrifuged $\left(2,300 \times \mathrm{g}, 4^{\circ} \mathrm{C}, 5 \mathrm{~min}\right)$. The water layer $(400 \mu \mathrm{L})$ was filtered through a 5-kDa-cutoff filter (ULTRAFREE-MCPLHCC; HMT, Yamagata, Japan) to remove macromolecules. The filtrate was centrifugally concentrated and resuspended in $50 \mu \mathrm{L}$ of ultrapure water immediately before measurement. The 
compounds were measured in the cation and anion modes of a capillary electrophoresis-Fourier transform mass spectrometry (CE-FTMS)-based metabolome analysis system under conditions $1-3$. The samples were diluted for the measurements to improve the quality of the CE-FTMS analysis. Metabolome measurements were conducted at HMT by using CE-time-of-flight mass spectrometry (TOFMS) in an Agilent CE System equipped with the Agilent 6210 time-of-flight mass spectrometer, the Agilent 1100 isocratic HPLC pump, the Agilent G1603A CEMS adapter kit, and the Agilent G1607A CE-ESI-MS sprayer kit (Agilent Technologies, Waldbronn, Germany). The system was controlled by Agilent G2201AA ChemStation software version B.03.01 for CE (Agilent Technologies, Waldbronn, Germany). The metabolites were analyzed using a fused silica capillary (50 $\mu \mathrm{m}$ internal diameter $\times 80 \mathrm{~cm}$ total length), with commercial electrophoresis buffers (Solution ID: H3301-1001 for cation analysis, H3302-1021 for anion analysis; HMT) as the electrolyte. The sample was injected at a pressure of 50 mbar for $10 \mathrm{~s}(\sim 10$ $\mathrm{nL})$ for cation analysis and $25 \mathrm{~s}(\sim 25 \mathrm{~nL})$ for anion analysis. The spectrometer was scanned from 50 to $1,000 \mathrm{~m} / \mathrm{z}$. Other conditions were as described previously (18-20). Peaks were extracted using the automatic integration software MasterHands (Keio University, Tsuruoka, Japan) to obtain peak information, including $\mathrm{m} / \mathrm{z}$, migration time for CE-TOFMS measurement (MT), and peak area values (21). Signal peaks corresponding to isotopomers, adduct ions, and other product ions of known metabolites were excluded, and the remaining peaks were annotated with putative metabolites from the HMT metabolite database based on their MTs and $\mathrm{m} / \mathrm{z}$ values, as determined from TOFMS. The tolerance range for the peak annotation was configured at $\pm 0.5 \mathrm{~min}$ for $\mathrm{MT}$ and $\pm 10 \mathrm{ppm}$ for $\mathrm{m} / \mathrm{z}$. In addition, peak areas were normalized against those of the internal standards, and the resultant relative areas were further normalized by the sample amount. Serum KynA levels were under limit of detection.

\section{Measurement of Serum Inflammatory Cytokine and BDNF Levels}

Samples were collected at the University of Occupational and Environmental Health and transferred to SRL Inc. (SRL: Kitakyushu, Japan). Serum cytokine (IL-1 $\beta$, IL-6, IL-10, TNF$\alpha)$ and BDNF levels were measured using the sandwich enzyme immunoassay technique. A monoclonal antibody specific for humans was pre-coated onto a microplate. Standards and samples were pipetted into the wells and any IL-1 $\beta$, IL-6, IL10 , TNF- $\alpha$, and BDNF present were bound by the immobilized antibody. After washing away any unbound substances, a biotinylated polyclonal antibody specific for humans was added to the wells. After a wash to remove any unbound antibodybiotin reagent, enzyme-linked streptavidin was added to the wells. After washing away any unbound streptavidin-enzyme reagent, a substrate solution was added to the wells and color developed in proportion to the amount of IL- $1 \beta$, IL6 , IL-10, TNF- $\alpha$, and BDNF bound in the initial step. Color development was stopped, and the intensity of the color was measured. Serum levels of IL-1 $\beta$, and IL-10 were under limit of detection.

\section{Measurement of Serum hsCRP Levels}

The high-sensitivity CRP (hsCRP) levels were measured using a commercially available enzyme-linked immunosorbent assay (MSD, Rockville, MD, USA).

\section{Statistical Analyses}

All statistical analyses were performed using the EZR software version 1.50, a modified version of $\mathrm{R}$ Commander designed to perform statistical functions frequently used in biostatistics (22). After confirming the normal distribution and equality of variance, continuous variables were analyzed using Student's $t$ test, Welch's test, and Mann-Whitney U test. Fisher's -test was used to analyze nominal variables. Spearman's rank correlation coefficient was used to examine the relationship among the levels of metabolites in the Kyn pathway, including their ratios (Trp, Kyn, 3-HK, QA, 5-hydroxytryptamine [5-HT], Kyn/Trp, 5-HT/Kyn, 3-HK/Kyn, QA/Kyn), and the levels of proinflammatory cytokines (TNF- $\alpha$, IL-6), hsCRP, BDNF and PANSS. The correlations between the data were expressed by correlation table. Positive correlations are shown in red, negative correlations are shown in blue, and the strength of the correlation is expressed in terms of concentration. A two-tailed test was used, and a $p$-value $<0.05$ was defined as a statistically significant difference. Dates were expressed mean(standard deviation) or

TABLE 1 | Clinical and demographic characteristics.

\begin{tabular}{|c|c|c|c|}
\hline & $\begin{array}{l}\text { Schizophrenia } \\
\qquad(n=30)\end{array}$ & $\begin{array}{l}\text { Healthy controls } \\
\qquad(n=10)\end{array}$ & $p$-value \\
\hline Age (years) & $48(9.0)$ & $48(8.7)$ & 0.99 \\
\hline Sex (males, \%) & 17 (57\%) & $5(50 \%)$ & 0.73 \\
\hline $\mathrm{BMl}\left(\mathrm{kg} / \mathrm{m}^{2}\right)$ & $23(3.8)$ & $23(2.8)$ & 0.89 \\
\hline \multicolumn{4}{|l|}{ PANSS } \\
\hline PANSS total & $95(13)$ & - & - \\
\hline PANSS positive & $21(4.6)$ & - & - \\
\hline PANSS negative & $26(4.9)$ & - & - \\
\hline PANSS general & $48(8.4)$ & - & - \\
\hline DIEPSS & $6.3(3.8)$ & - & - \\
\hline CP total & 745 (460) & - & - \\
\hline Disease period & $26(10)$ & - & - \\
\hline \multirow[t]{12}{*}{ Antipsychotic drugs } & Olanzapine (6 cases) & & \\
\hline & Risperidone (6 cases) & & \\
\hline & Levomepromazine (5 cases) & & \\
\hline & Aripiprazole (5 cases) & & \\
\hline & Clozapine (5 cases) & & \\
\hline & Haloperidol (4 cases) & & \\
\hline & Brexpiprazole (3 cases) & & \\
\hline & Quetiapine (3 cases) & & \\
\hline & Zotepine (3 cases) & & \\
\hline & Asenapine (2 cases) & & \\
\hline & Blonanserin (2 cases) & & \\
\hline & Fluphenazine (1 cases) & & \\
\hline
\end{tabular}

BMI, body mass index; PANSS, Positive and Negative Syndrome Scale; DIEPSS, Drug Induced Extra-Pyramidal Symptoms Scale; CP total, total dose of chlorpromazine equivalence. Dates were expressed mean(standard deviation). 
TABLE 2 | Serum levels of the metabolites of the Kyn pathway in the schizophrenia and healthy control groups.

\begin{tabular}{|c|c|c|c|}
\hline & Schizophrenia $(n=30)$ & Healthy controls $(n=10)$ & $p$-value \\
\hline Trp & $1.9 \times 10^{-1}\left(4.5 \times 10^{-2}\right)$ & $2.1 \times 10^{-1}\left(3.1 \times 10^{-2}\right)$ & 0.11 \\
\hline Kyn & $6.0 \times 10^{-3}\left(2.0 \times 10^{-3}\right)$ & $6.9 \times 10^{-3}\left(1.3 \times 10^{-3}\right)$ & 0.26 \\
\hline $3-\mathrm{HK}$ & $5.6 \times 10^{-5}\left[4.3 \times 10^{-5} \sim 8.0 \times 10^{-5}\right]$ & $7.1 \times 10^{-5}\left[5.8 \times 10^{-5} \sim 1.0 \times 10^{-4}\right]$ & 0.052 \\
\hline QA & $8.6 \times 10^{-5}\left[6.6 \times 10^{-5} \sim 1.4 \times 10^{-4}\right]$ & $1.1 \times 10^{-4}\left[4.6 \times 10^{-5} \sim 1.4 \times 10^{-4}\right]$ & 0.99 \\
\hline $5-\mathrm{HT}$ & $1.1 \times 10^{-3}\left(5.3 \times 10^{-4}\right)$ & $1.0 \times 10^{-3}\left(6.8 \times 10^{-4}\right)$ & 0.80 \\
\hline Kyn/Try & $0.03[0.02 \sim 0.06]$ & 0.03 [0.02 0.05] & 0.93 \\
\hline 5HT/Kyn & $0.17[0.094 \sim 0.25]$ & $0.12[0.11 \sim 0.20]$ & 0.57 \\
\hline 3-HK/Kyn & $0.01[0.01 \sim 0.10]$ & $0.01[0.01 \sim 0.02]$ & 0.97 \\
\hline QA/Kyn & 0.01 [0.01 0.02] & 0.01 [0.008 0.02] & 0.98 \\
\hline
\end{tabular}

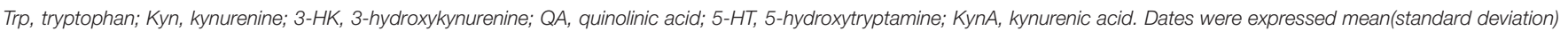

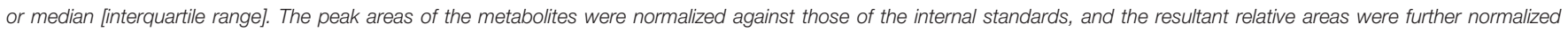

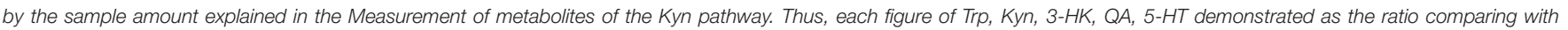
internal standard.

TABLE 3 | Serum levels of cytokines, hsCRP, and BDNF in the schizophrenia group and the healthy control.

\begin{tabular}{lccc}
\hline & Schizophrenia $(\boldsymbol{n}=\mathbf{3 0})$ & Healthy controls $(\boldsymbol{n}=10)$ & $\boldsymbol{p}$-value \\
\hline $\mathrm{IL}-6(\mathrm{pg} / \mathrm{mL})$ & $1.5[0.9 \sim 2.1]$ & $1.1[0.6 \sim 2.0]$ & 0.23 \\
$\mathrm{TNF}-\alpha(\mathrm{pg} / \mathrm{mL})$ & $0.9[0.7 \sim 1.0]$ & $0.9[0.7 \sim 0.9]$ & 0.52 \\
$\mathrm{hsCRP}(\mathrm{mg} / \mathrm{dL})$ & $0.029[0.0070 \sim 0.065]$ & $0.030[0.017 \sim 0.065]$ & 0.80 \\
$\mathrm{BDNF}(\mathrm{pg} / \mathrm{mL})$ & $22,000(7,800)$ & $22,000(5,000)$ & 0.99
\end{tabular}

hsCRP, high-sensitivity C-reactive protein; BDNF, brain-derived neurotrophic factor; IL, interleukin; TNF- $\alpha$, tumor necrosis factor alpha. Dates were expressed mean(standard deviation) or median [interquartile range].

median [interquartile range]. Figures that showed statistically significant differences in the correlation table were drawn. The regression line was drawn based on the least squares method. One schizophrenia patient had outlier data for CRP $(7.2 \mathrm{mg} / \mathrm{dL})$ and IL-6 (24 pg/mL) levels; therefore, we excluded these data.

\section{RESULTS}

\section{Background Characteristics}

Thirty patients with schizophrenia and ten healthy participants were included in this study. The background and clinical characteristics of the patients with schizophrenia and healthy controls are shown (Table 1). No significant difference was observed in the background characteristics between patients with schizophrenia and healthy controls.

\section{Serum Levels of the Metabolites of the Kyn Pathway in the Schizophrenia and Healthy Control Groups}

We evaluated the serum levels of metabolites of the Kyn pathway (Trp, Kyn, 3-HK, QA, 5-HT, Kyn/Trp, 5-HT/Kyn, 3$\mathrm{HK} / \mathrm{Kyn}, \mathrm{QA} / \mathrm{Kyn}$ ) in patients with schizophrenia and healthy controls. No significant differences were observed in the levels of the metabolites of the Kyn pathway between patients with schizophrenia and healthy controls (Table 2).

\section{Serum Levels of Cytokines, hsCRP, and BDNF in the Schizophrenia and Healthy Control Groups}

We also evaluated the serum levels of cytokines (TNF- $\alpha$, IL-6), hsCRP, and BDNF in patients with schizophrenia and healthy controls (Table 3). No significant difference was observed in serum levels of the cytokines (TNF- $\alpha$, IL-6), hsCRP, and BDNF between the schizophrenia and healthy control groups. IL-1 $\beta$ and IL-10 were not detected in the samples.

\section{Relationships Among Metabolites of the Kyn Pathway and Cytokines, hsCRP, and BDNF in the Schizophrenia and Healthy Control Groups}

The correlation table showed the relationships between the Kyn pathway metabolites and the cytokines (TNF- $\alpha$, IL-6), hsCRP, and BDNF in the schizophrenia group and the healthy control group (Table 4). A significant positive correlation was found between the serum TNF- $\alpha$ levels and the serum Kyn levels $(r$ $=0.53, p=0.0026$, Figure 1) and the Kyn/Trp value $(r=$ 0.67, $p=0.000046$, Figure 2) in the schizophrenia group. A significant positive correlation was found between the serum level of BDNF and the serum level of 5-HT $(r=0.49, p=$ 0.0061 , Figure 3$)$ and the $5-\mathrm{HT} / \mathrm{Trp}$ value $(r=0.55, p=0.0018$, Figure 4) in the schizophrenia group (Table 4A). A positive correlation was found between the serum levels of IL-6 and the serum levels of Kyn $(r=0.64, p=0.046$, Figure 5) and QA $(r=0.78, p=0.010$, Figure 6) as well as the QA/Kyn value $(r=0.77, p=0.021$, Figure 7$)$ in the healthy control group (Table 4B).

\section{Relationship Between the PANSS Scores and the Serum Levels of Metabolites of the Kyn Pathway, Cytokines, hsCRP, and BDNF in the Schizophrenia Group}

The correlation table showed the relationship between the PANSS scores and the serum levels of metabolites of the Kyn pathway and BDNF (Table 5). A negative correlation was found between 
TABLE 4A | Relationship between metabolites of the Kyn pathway and cytokines, hsCRP, and BDNF in the schizophrenia group.

\begin{tabular}{|c|c|c|c|c|}
\hline & IL-6 & TNF- $\alpha$ & hsCRP & BDNF \\
\hline \multirow[t]{2}{*}{ Trp } & 0.028 & -0.092 & 0.33 & 0.087 \\
\hline & $(p=0.89)$ & $(p=0.63)$ & $(p=0.080)$ & $(p=0.65)$ \\
\hline \multirow[t]{2}{*}{ Kyn } & 0.3 & 0.53 & 0.36 & -0.21 \\
\hline & $(p=0.11)$ & $(p=0.0026)$ & $(p=0.051)$ & $(p=0.26)$ \\
\hline \multirow[t]{2}{*}{ 3-HK } & 0.24 & 0.29 & 0.25 & -0.21 \\
\hline & $(p=0.21)$ & $(p=0.13)$ & $(p=0.18)$ & $(p=0.28)$ \\
\hline \multirow[t]{2}{*}{ QA } & 0.42 & 0.34 & 0.27 & -0.23 \\
\hline & $(p=0.059)$ & $(p=0.12)$ & $(p=0.24)$ & $(p=0.31)$ \\
\hline \multirow[t]{2}{*}{ 5-HT } & -0.3 & -0.032 & -0.23 & 0.49 \\
\hline & $(p=0.13)$ & $(p=0.87)$ & $(p=0.23)$ & $(p=0.0061)$ \\
\hline \multirow[t]{2}{*}{ Kyn/Trp } & 0.27 & 0.67 & 0.18 & -0.26 \\
\hline & $(p=0.16)$ & $(p=0.000046)$ & $(p=0.36)$ & $(p=0.16)$ \\
\hline \multirow[t]{2}{*}{ 5-HT/Kyn } & -0.34 & 0.1 & -0.24 & 0.55 \\
\hline & $(p=0.069)$ & $(p=0.60)$ & $(p=0.22)$ & $(p=0.0018)$ \\
\hline \multirow[t]{2}{*}{ 3-HK/Kyn } & 0.063 & 0.015 & 0.1 & -0.12 \\
\hline & $(p=0.75)$ & $(p=0.93)$ & $(p=0.60)$ & $(p=0.52)$ \\
\hline \multirow[t]{2}{*}{ QA/Kyn } & 0.32 & 0.25 & 0.16 & 0.0085 \\
\hline & $(p=0.16)$ & $(p=0.27)$ & $(p=0.50)$ & $(p=0.97)$ \\
\hline
\end{tabular}

TABLE 4B | Relationship between metabolites of the Kyn pathway and cytokines, hsCRP, and BDNF in the healthy control group.

\begin{tabular}{|c|c|c|c|c|}
\hline & IL-6 & TNF- $\alpha$ & hsCRP & BDNF \\
\hline \multirow[t]{2}{*}{ Trp } & -0.12 & 0.25 & 0.28 & 0.056 \\
\hline & $(p=0.74)$ & $(p=0.49)$ & $(p=0.44)$ & $(p=0.87)$ \\
\hline \multirow[t]{2}{*}{ Kyn } & 0.64 & 0.61 & 0.33 & -0.25 \\
\hline & $(p=0.046)$ & $(p=0.060)$ & $(p=0.35)$ & $(p=0.49)$ \\
\hline \multirow[t]{2}{*}{$3-\mathrm{HK}$} & -0.055 & -0.15 & 0.091 & 0.29 \\
\hline & $(p=0.89)$ & $(p=0.68)$ & $(p=0.81)$ & $(p=0.42)$ \\
\hline \multirow[t]{2}{*}{ QA } & 0.78 & 0.69 & 0.58 & -0.071 \\
\hline & $(p=0.010)$ & $(p=0.040)$ & $(p=0.10)$ & $(p=0.86)$ \\
\hline \multirow[t]{2}{*}{ 5-HT } & -0.33 & 0.45 & 0.46 & 0.073 \\
\hline & $(p=0.35)$ & $(p=0.20)$ & $(p=0.18)$ & $(p=0.84)$ \\
\hline \multirow[t]{2}{*}{ Kyn/Trp } & 0.55 & 0.46 & 0.22 & -0.18 \\
\hline & $(p=0.10)$ & $(p=0.18)$ & $(p=0.53)$ & $(p=0.62)$ \\
\hline \multirow[t]{2}{*}{ 5-HT/Kyn } & -0.35 & 0.37 & 0.50 & -0.054 \\
\hline & $(p=0.33)$ & $(p=0.30)$ & $(p=0.15)$ & $(p=0.88)$ \\
\hline \multirow[t]{2}{*}{ 3-HK/Kyn } & -0.055 & -0.15 & 0.091 & 0.29 \\
\hline & $(p=0.89)$ & $(p=0.68)$ & $(p=0.81)$ & $(p=0.42)$ \\
\hline \multirow[t]{2}{*}{ QA/Kyn } & 0.77 & 0.63 & 0.58 & -0.075 \\
\hline & $(p=0.021)$ & $(p=0.070)$ & $(p=0.11)$ & $(p=0.84)$ \\
\hline
\end{tabular}

hsCRP, high-sensitivity C-reactive protein; BDNF, brain-derived neurotrophic factor; IL, interleukin; TNF- $\alpha$, tumor necrosis factor alpha; Trp, tryptophan; Kyn, kynurenine; 3-HK, 3hydroxykynurenine; QA, quinolinic acid; 5-HT, 5-hydroxytryptamine; KynA, kynurenic acid. Statistically significant differences at $P<0.05$ is in bold. Positive correlations are shown in red, negative correlations are shown in blue, and the strength of the correlation is expressed in terms of concentration.

the PANSS-G score and the serum QA levels $(r=-0.44, p$ $=0.043$, Figure 8) and the QA/Kyn value $(r=-0.64, p=$ 0.0014 , Figure 9). A negative correlation was found between the

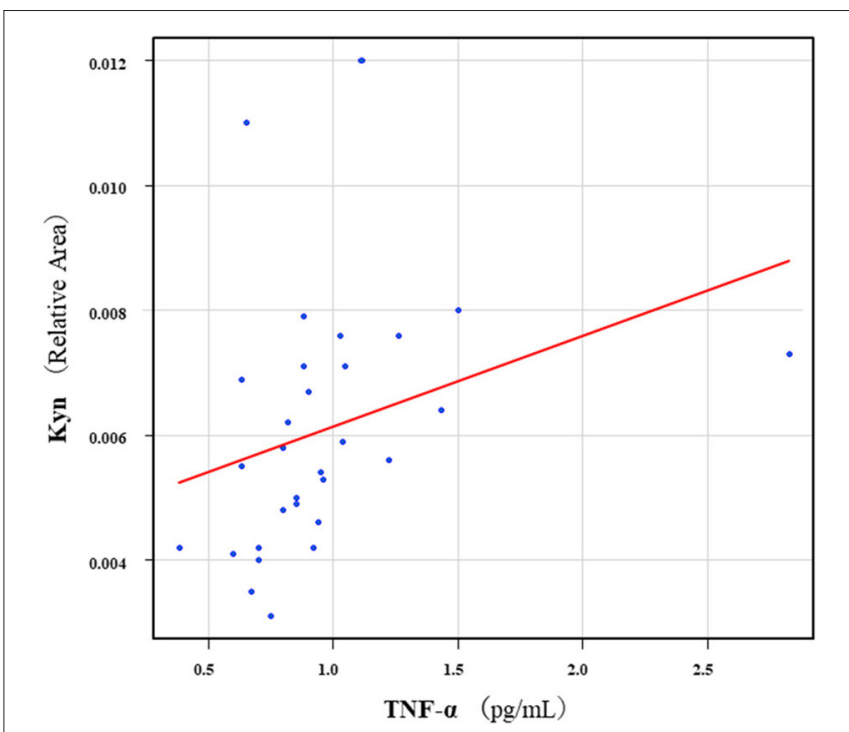

FIGURE 1 | Association between serum TNF- $\alpha$ levels and serum Kyn levels in the schizophrenia group. A significant positive correlation was found between the serum TNF- $\alpha$ levels and the serum Kyn levels ( $r=0.53, p=0.0026$ ). Relative Area: The peak areas of the metabolites were normalized against those of the internal standards, and the resultant relative areas were further normalized by the sample amount explained in the Measurement of metabolites of the Kyn pathway. Thus, serum Kyn levels demonstrated as the ratio comparing with internal standard.

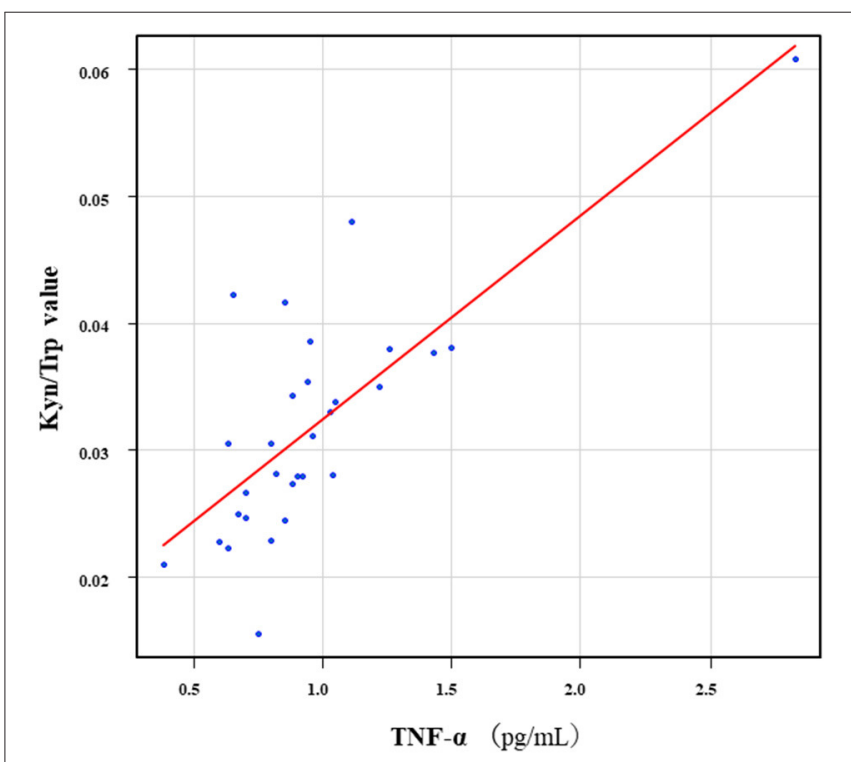

FIGURE 2 | Association between serum TNF- $\alpha$ levels and the Kyn/Trp value in the schizophrenia group. A significant positive correlation was found between the serum TNF- $\alpha$ levels and the and the Kyn/Trp value $(r=0.67, p=$ 0.000046 ) in the schizophrenia group.

serum level of BDNF and the PANSS-N score $(r=-0.38, p=$ 0.038 , Figure 10). A negative correlation was found between the 


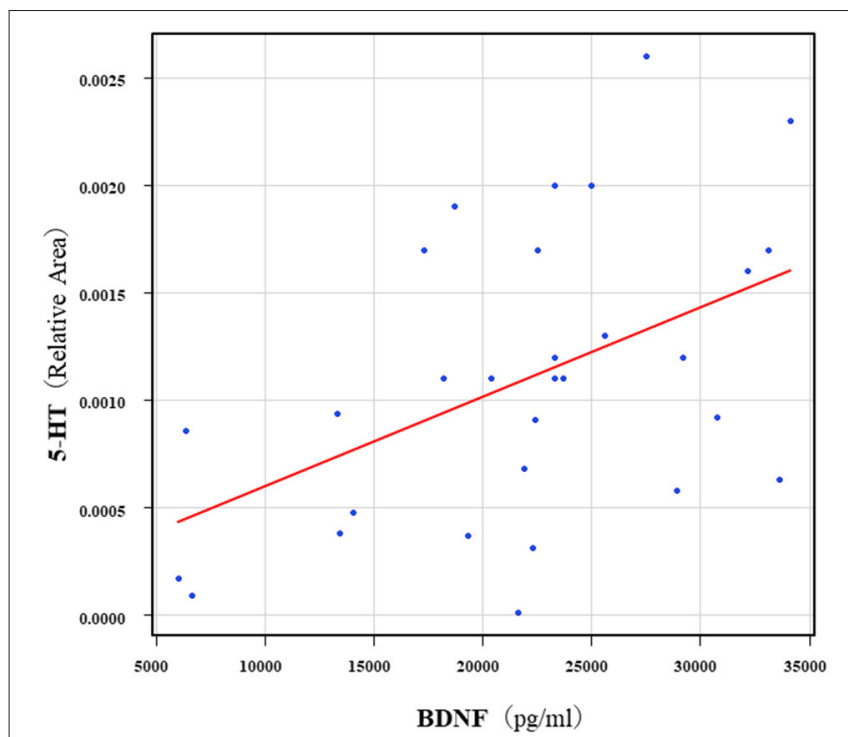

FIGURE 3 | Association between serum 5-HT levels and serum BDNF levels in the schizophrenia group 5. A significant positive correlation was found between the serum BDNF level and the serum 5-HT levels $(r=0.49, p=$ $0.0061)$ in the schizophrenia group. Relative Area: The peak areas of the metabolites were normalized against those of the internal standards, and the resultant relative areas were further normalized by the sample amount explained in the Measurement of metabolites of the Kyn pathway. Thus, serum 5-HT levels demonstrated as the ratio comparing with internal standard.

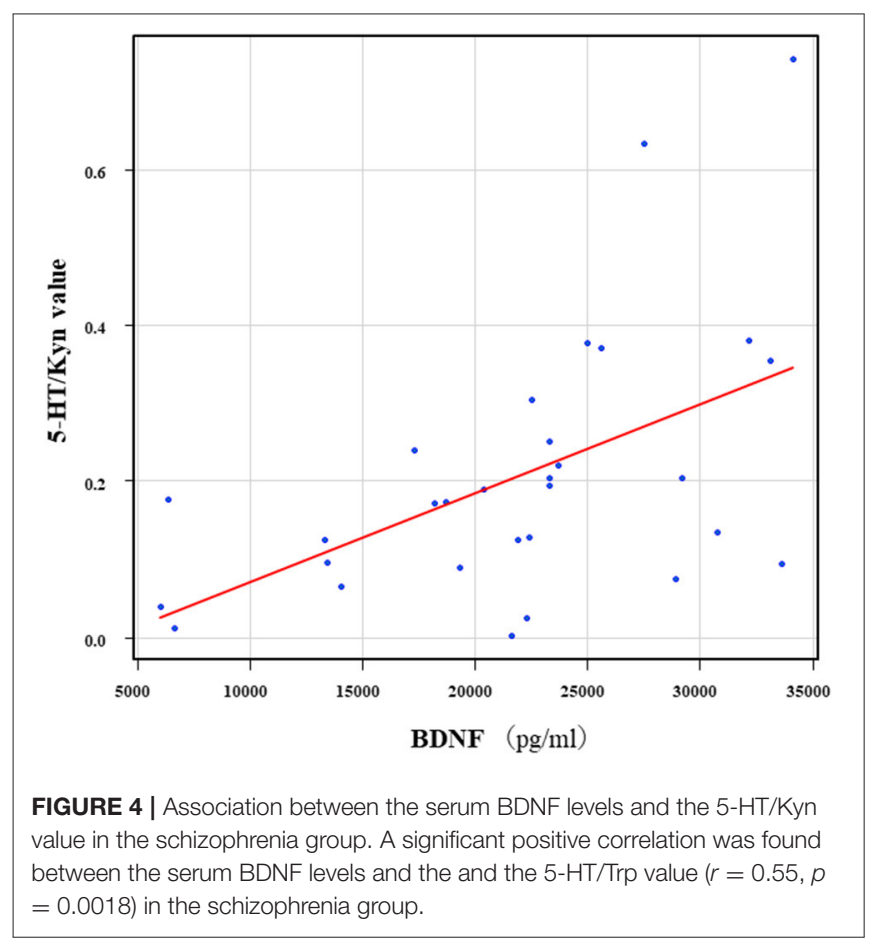

PANSS-T score and the QA/Kyn value $(r=-0.50, p=0.017$, Figure 11).
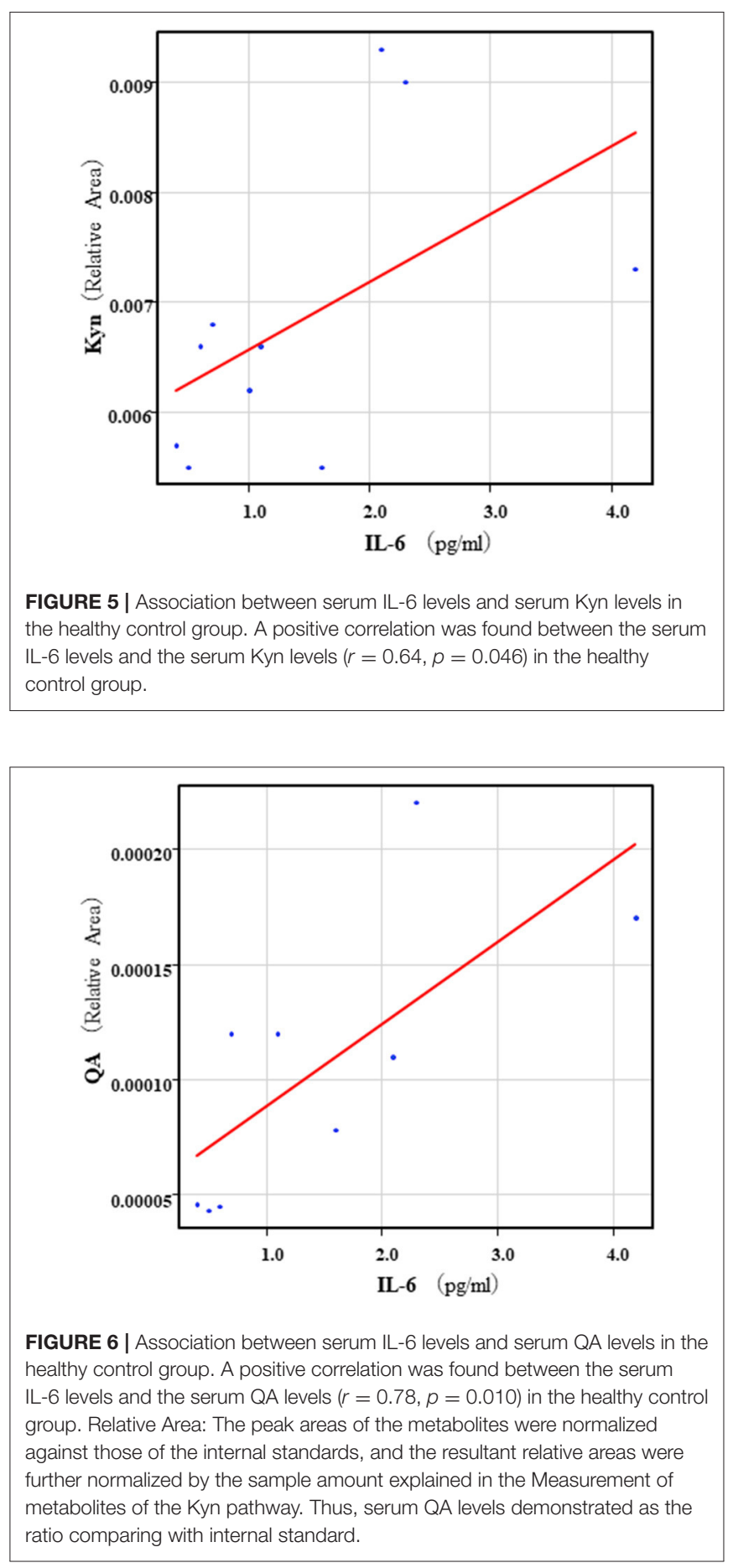

\section{DISCUSSION}

Our findings showed a significant positive correlation between the serum levels of TNF- $\alpha$ and the Kyn levels and the Kyn/Trp value in the schizophrenia group, but not in the healthy control group. These results suggest that TNF- $\alpha$ accelerates the formation of Kyn from Trp in patients with schizophrenia. Inflammatory cytokines, especially TNF- $\alpha$ and IL-6, are the primary molecular 


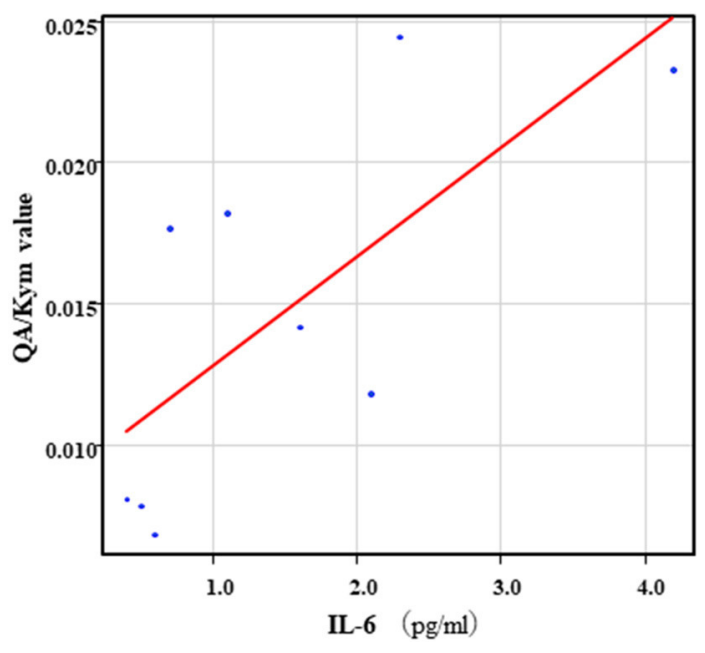

FIGURE 7 | Association between serum IL-6 levels and serum QA/Kyn value in the healthy control group. A positive correlation was found between the serum levels of IL-6 and the serum levels of QA/Kyn value $(r=0.77, p=$ 0.021 ) in the healthy control group. targets for schizophrenia. Within central nervous system, microglia, the enzyme indoleamine 2,3-dioxygenase plays a role in the metabolism of Trp to Kyn and the subsequent conversion of Kyn to neurotoxic QA. At the same time, cytokine activation shunts metabolic activity from Trp to the Kyn pathway, which further reduces tryptophan hydroxylase driven serotonin synthesis (23). Serum levels of TNF- $\alpha$ in the schizophrenia group were not different from those in the healthy control group in the present study. Thus, it is possible that TNF- $\alpha$, but not IL-6 more potently activates indoleamine 2,3dioxygenase and tryptophan 2,3-dioxygenas, two major ratelimiting enzymes of Kyn formation in the schizophrenia group than in the healthy control group. Exposure to chronic mild stress (24), or hepatic encephalopathy (25) has been shown to increase TNF- $\alpha$ and indoleamine 2,3-dioxygenase activity in rats. The serum levels of TNF- $\alpha$ are elevated in schizophrenia $(6,26)$. Serum levels of IL-6 (26-28), but not the cerebrospinal fluid levels of IL-6 (29), were also elevated in schizophrenia patients. Serum levels of QA were also elevated in psychiatric controls (30). QA, an endogenous metabolite of the Kyn pathway, is toxic and is involved in several neuropsychiatric diseases, including schizophrenia $(7,31)$. Taken together, these results suggest that IL-6 combined with QA might contribute to neuronal damage in the brain in schizophrenia. A recent systematic review (3) examined the correlation between cytokines and Kyn metabolites, and three studies showed a relationship between the Kyn pathway and elevated IL- 6 and TNF- $\alpha$ concentrations. Only one study showed correlations between IL- 8 concentrations and the Kyn pathway, and two studies showed correlations of low IL4 concentrations with the Kyn pathway. Moreover, the authors of the systematic review did not find significant correlations of CRP ( $n=1$ study) and IFN- $\gamma$ ( $n=3$ studies) with the Kyn pathway in schizophrenia. Meta-analyses of CRP levels in
TABLE 5 | Relationship between metabolites of the Kyn pathway and positive and negative syndrome scale scores.

\begin{tabular}{|c|c|c|c|c|}
\hline & PANSS-T & PANSS-P & PANSS-N & PANSS-G \\
\hline \multirow[t]{2}{*}{ Trp } & -0.16 & 0.0021 & 0.0019 & -0.35 \\
\hline & $(p=0.40)$ & $(p=0.99)$ & $(p=0.99)$ & $(p=0.06)$ \\
\hline \multirow[t]{2}{*}{ Kyn } & -0.17 & -0.054 & -0.039 & -0.28 \\
\hline & $(p=0.38)$ & $(p=0.78)$ & $(p=0.84)$ & $(p=0.14)$ \\
\hline \multirow[t]{2}{*}{ 3-HK } & -0.08 & 0.12 & -0.044 & -0.13 \\
\hline & $(p=0.68)$ & $(p=0.53)$ & $(p=0.82)$ & $(p=0.50)$ \\
\hline \multirow[t]{2}{*}{ QA } & -0.3 & -0.21 & -0.15 & -0.44 \\
\hline & $(p=0.16)$ & $(p=0.35)$ & $(p=0.45)$ & $(p=0.043)$ \\
\hline \multirow[t]{2}{*}{$5-\mathrm{HT}$} & 0.19 & 0.099 & 0.1 & 0.18 \\
\hline & $(p=0.33)$ & $(p=0.60)$ & $(p=0.58)$ & $(p=0.33)$ \\
\hline \multirow[t]{2}{*}{ Kyn/Trp } & -0.025 & -0.0078 & -0.028 & -0.0045 \\
\hline & $(p=0.89)$ & $(p=0.97)$ & $(p=0.88)$ & $(p=0.98)$ \\
\hline \multirow[t]{2}{*}{ 5HT/Kyn } & 0.22 & 0.13 & 0.067 & 0.27 \\
\hline & $(p=0.25)$ & $(p=0.51)$ & $(p=0.73)$ & $(p=0.14)$ \\
\hline \multirow[t]{2}{*}{ 3-HK/Kyn } & 0.078 & 0.27 & 0.014 & 0.048 \\
\hline & $(p=0.68)$ & $(p=0.15)$ & $(p=0.94)$ & $(p=0.80)$ \\
\hline \multirow[t]{2}{*}{ QA/Kyn } & -0.5 & -0.38 & -0.24 & -0.64 \\
\hline & $(p=0.017)$ & $(p=0.076)$ & $(p=0.28)$ & $(p=0.0014)$ \\
\hline \multirow[t]{2}{*}{ IL-6 } & -0.33 & -0.2 & -0.28 & -0.29 \\
\hline & $(p=0.080)$ & $(p=0.30)$ & $(p=0.14)$ & $(p=0.13)$ \\
\hline \multirow[t]{2}{*}{ TNF- $\alpha$} & -0.24 & -0.2 & -0.13 & -0.21 \\
\hline & $(p=0.21)$ & $(p=0.30)$ & $(p=0.49)$ & $(p=0.26)$ \\
\hline \multirow[t]{2}{*}{ hsCRP } & -0.26 & -0.28 & -0.04 & -0.29 \\
\hline & $(p=0.18)$ & $(p=0.15)$ & $(p=0.84)$ & $(p=0.13)$ \\
\hline \multirow[t]{2}{*}{ BDNF } & -0.34 & -0.21 & -0.38 & -0.23 \\
\hline & $(p=0.064)$ & $(p=0.26)$ & $(p=0.038)$ & $(p=0.22)$ \\
\hline
\end{tabular}

PANSS, Positive and Negative Syndrome Scale; PANSS-T, PANSS Total; PANSSP, PANSS Positive; PANSS-N, PANSS Negative; PANSS-G, PANSS General; Trp, tryptophan; Kyn, kynurenine; 3-HK, 3-hydroxykynurenine; QA, quinolinic acid; 5HT, 5-hydroxytryptamine; KynA, kynurenic acid; hsCRP, high-sensitivity C-reactive protein; BDNF, brain-derived neurotrophic factor; IL, interleukin; TNF- $\alpha$, tumor necrosis factor alpha. Statistically significant differences at $P<0.05$ is in bold. Positive correlations are shown in red, negative correlations are shown in blue, and the strength of the correlation is expressed in terms of concentration.

schizophrenia, which included a total of 26 cross-sectional or longitudinal studies evaluating 85,000 participants demonstrated that CRP levels were moderately increased (7). We found a positive correlation between serum levels of hsCRP and serum levels of Kyn in the schizophrenia group. Furthermore, a positive correlation was found between serum levels of BDNF and serum levels of 5-HT in the schizophrenia group, but not in the healthy control group. The 5-HT and BDNF identified in the serum were mainly secreted by platelets $(32,33)$. The discrepancies between the schizophrenia and healthy control groups might reflect differences in the secretory activity of platelets in both groups. In other words, the interaction between BDNF and 5-HT may be tighter in the schizophrenia group. However, the precise reasons underlying these results remain unknown.

A negative correlation was found between PANSS scores and the serum levels of QA and the QA/Kyn ratio in the schizophrenia group. However, when QA has neurotoxic effects, 


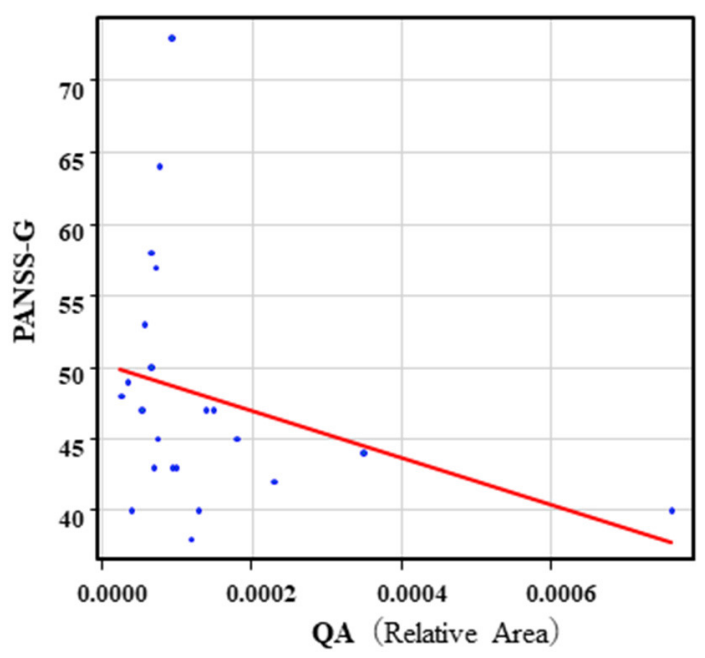

FIGURE 8 | Association between the PANSS-G scores and serum QA levels in the schizophrenia group. A negative correlation was found between the PANSS-G score and serum QA levels $(r=-0.44, p=0.043)$ in the schizophrenia group. Relative Area: The peak areas of the metabolites were normalized against those of the internal standards, and the resultant relative areas were further normalized by the sample amount explained in the Measurement of metabolites of the Kyn pathway. Thus, serum QA levels demonstrated as the ratio comparing with internal standard.

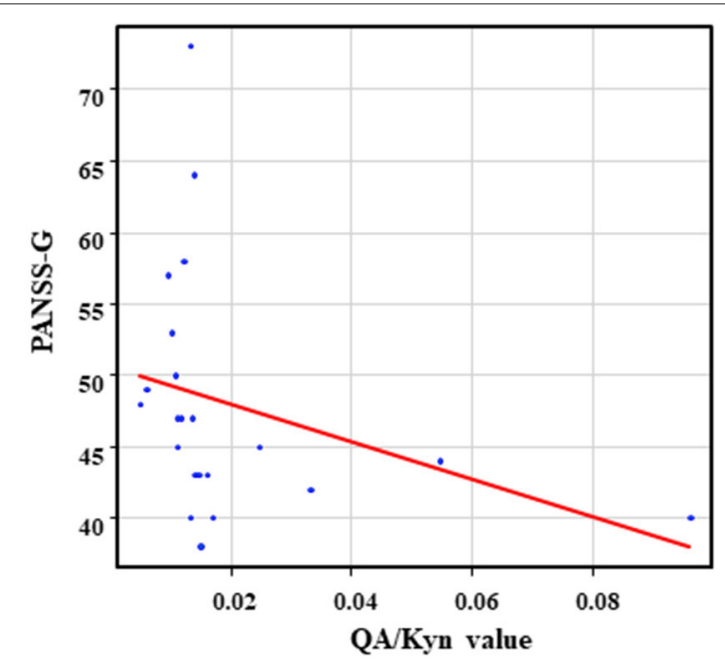

FIGURE 9 | Association between the PANSS-G scores and the QA/Kyn value in the schizophrenia group. A negative correlation was found between the PANSS-G score and the QA/Kyn value $(r=-0.64, p=0.0014)$ in the schizophrenia group.

a positive correlation has been reported be found between PANSS scores and the QA level or QA/Kyn ratio. This discrepancy in the present results was difficult to interpret. QA shows cellular neurotoxicity and has implications in schizophrenia. It has been speculated an imbalance in the production or removal of either of Kyn and QA would be expected to have profound implications for brain function, especially if that

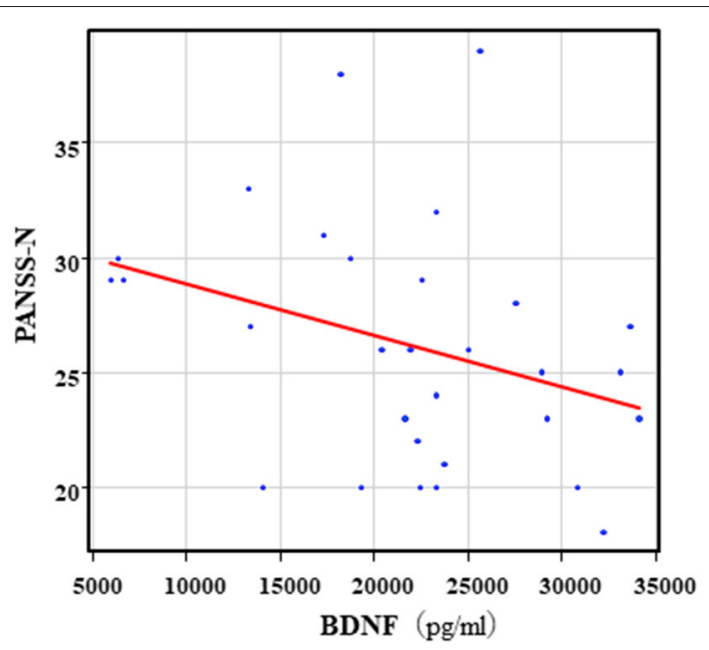

FIGURE 10 | Association between the PANSS-N scores and serum BDNF levels in the schizophrenia group. A negative correlation was found between he PANSS-N score and the serum BDNF levels $(r=-0.38, p=0.038)$ in the schizophrenia group.

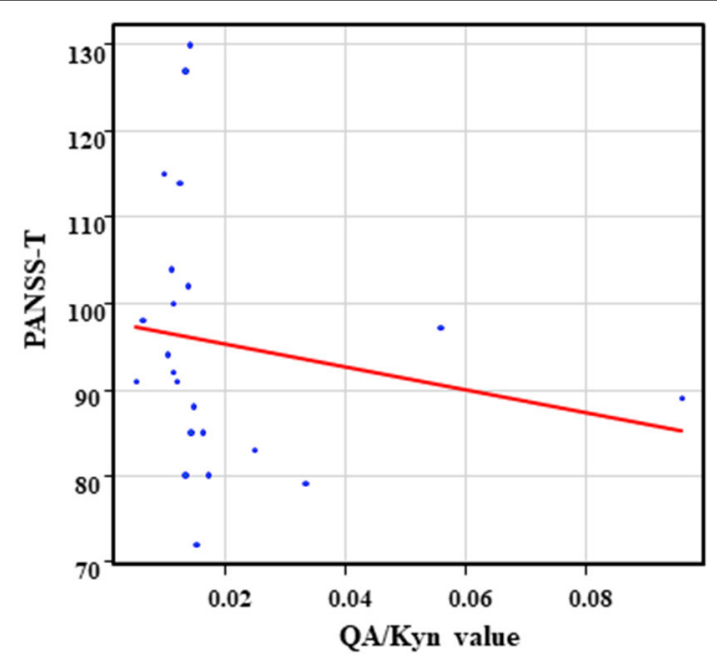

FIGURE 11 | Association between the PANSS-T scores and the QA/Kyn value in the schizophrenia group. A negative correlation was found between the PANSS-T sore and the QA/Kyn value $(r=-0.50, p=0.017)$ in the schizophrenia group.

imbalance were present chronically (34). Recent study reviewed the roles of imbalances in Kyn metabolism in association with interactions with other neurochemicals as a major contributing pathophysiological mechanism in schizophrenia (35). Actually, QA was correlated with BDNF levels and psychiatric symptoms, suggesting that QA may have an indirect effect on psychiatric symptoms through BDNF but may also have a direct effect simultaneously. This may be biologically plausible because QA is an NMDA receptor agonist that can additionally inhibit the reuptake of glutamate by astrocytes, leading to excitotoxicity $(7,31)$. We also found a positive correlation between serum 
QA and serum IL-6 levels in the schizophrenia and healthy control groups. Negative correlations were found between the PANSS-T scores and serum levels of IL-6 and between the PANSS-G scores and serum levels of BDNF in the schizophrenia group. A recent meta-analysis demonstrated that peripheral BDNF levels in serum and plasma were moderately reduced in patients with schizophrenia compared with controls and that this decrease was associated with disease duration. The extent of peripheral BDNF level decrease, however, did not correlate with the severity of positive and negative symptoms (4). Serum levels of IL-6 in chronic schizophrenia patients at admission showed a positive correlation with negative scores, and the serum levels of IL-6 in the patients at discharge were positively correlated with positive, negative, and total scores (36). These findings suggest that the association of serum levels of BDNF and IL-6 with psychometric findings might be complicated in schizophrenia patients. Previous studies have reported that the Kyn pathway is promoted by inflammation, which could be related to the pathological organization of many psychiatric disorders, including schizophrenia. BDNF is a protein produced by nerve cells in the brain and plays an important role in nerve cell activity. Several studies have reported serum Kyn levels in schizophrenia; however, there is no consistent view because of the many conflicting results. Our results showed that there was no significant difference in the plasma levels of Kyn pathway metabolites, cytokines, and BDNF between the schizophrenia and healthy control groups. A partial downregulation of the Kyn pathway is observed in schizophrenia patients, especially during acute symptomatic states and in older age, effects that are independent of each other. In contrast, younger and stable or remitted patients display limited to no Kyn metabolite abnormalities. The current meta-analysis illustrates the dynamic nature of Kyn abnormalities. It should be noted that all included studies investigated peripheral Kyn metabolites, which do not necessarily reflect central Kyn metabolite abnormalities in patients with schizophrenia (37). Finally, the blood levels of metabolites of the Kyn pathway, proinflammatory cytokines, hsCRP, and BDNF in patients with chronic schizophrenia remain controversial. Their interactions may also be complicated. The reasons for this discrepancy in the results remain unknown; however, the diversity of schizophrenia patients enrolled in past and current studies is a plausible explanation.

It has been reported that a positive correlation in Kyn, Trp, or Kyn/Trp ratio between serum and CSF (38), On the other hand, a discrepancy existed in cytokines between serum and CSF (39). Finally, the parallel changes in BDNF levels in plasma and CSF indicate that plasma BDNF levels reflect the brain changes in BDNF levels in schizophrenia (40). The definite discrepancy exists in metabolites of Kyn pathway, cytokines, or BDNF between the peripheral and the brain. Taken together, we could not interpret the results in peripheral as in the brain.

It has been speculated that antipsychotic drugs influence the upregulated Kyn pathway in schizophrenia patients (41).
In contrast, treatment of haloperidol and clozapine did not affect the levels of brain Kyn or KynA in mice (9). The precise mechanisms how antipsychotic drugs influence the Kyn pathway and merge their efficacies remain unknown, this must be further elucidated.

This study had several limitations. First, the sample size was small, and all patients with schizophrenia were receiving several antipsychotic drugs, which could have affected the serum Kyn and Kyn metabolite levels. Second, we could not measure KynA in the Kyn pathway, IL-10 and IL-1 $\beta$. Third, Trp is an essential amino acid that is consumed through diet, and aerobatic exercise but this study did not consider nutritional status, and daily activities of the schizophrenia patients.

In conclusion, TNF- $\alpha$ could influence the Kyn pathway in chronic hospitalized patients with schizophrenia. The relationships of metabolites of the Kyn pathway with the levels of proinflammatory cytokines, hsCRP, and BDNF and psychotic symptoms in chronic schizophrenia are complicated and must be further elucidated.

\section{DATA AVAILABILITY STATEMENT}

The original contributions presented in the study are included in the article/supplementary material, further inquiries can be directed to the corresponding author.

\section{ETHICS STATEMENT}

The studies involving human participants were reviewed and approved by Ethics Committee of the University of Occupational and Environmental Health (Approval Number: UOEHCRB19024). The patients/participants provided their written informed consent to participate in this study.

\section{AUTHOR CONTRIBUTIONS}

NO and RY: conceptualization. NO: methodology, software, and visualization. NO, YK, AI, and RY: validation and writingoriginal draft preparation. NO, HT, TN, RI, YK, and AI: data curation. AI, YK, and RY: writing-review, editing, and supervision. RY: funding acquisition. All authors have read and agreed to the published version of the manuscript.

\section{ACKNOWLEDGMENTS}

The authors gratefully thank Ms. Tomomi Ishiba for their anytime help with blood sampling and paperwork. The authors also thank Dr. Koji Yoshino, Ms. Chinami Moriyasu, Ms. Aya Yoshida, Mr. Takeharu Iwasaki and Ms. Tomoe Iwasaki (Komine-Eto Hospital, Kitakyushu, Japan) for their help with experimental procedures and thank other medical institutions that contributed to our research. 


\section{REFERENCES}

1. Müller N, Weidinger E, Leitner B, Schwarz MJ. The role of inflammation in schizophrenia. Front Neurosci. (2015) 9:372. doi: 10.3389/fnins.2015.00372

2. Buckley PF. Neuroinflammation and schizophrenia. Curr Psychiatry Rep. (2019) 21:1-3. doi: 10.1007/s11920-019-1050-z

3. Pedraz-Petrozzi B, Elyamany O, Rummel C, Mulert C. Effects of inflammation on the kynurenine pathway in schizophrenia-a systematic review. $J$ Neuroinflammation. (2020) 17:1-7. doi: 10.1186/s12974-020-1721-Z

4. Fernandes BS, Steiner J, Berk M, Molendijk ML, Gonzalez-Pinto A, Turck $\mathrm{CW}$, et al. Peripheral brain-derived neurotrophic factor in schizophrenia and the role of antipsychotics: meta-analysis and implications. Mol Psychiatry. (2015) 20:1108-19. doi: 10.1038/mp.2014.117

5. Qin XY, Wu HT, Cao C, Loh YP, Cheng Y. A meta-analysis of peripheral blood nerve growth factor levels in patients with schizophrenia. Mol Psychiatry. (2017) 22:1306-12. doi: 10.1038/mp.2016.235

6. Goldsmith DR, Rapaport MH, Miller BJ. A meta-analysis of blood cytokine network alterations in psychiatric patients: comparisons between schizophrenia, bipolar disorder and depression. Mol Psychiatry. (2016) 21:1696-709. doi: 10.1038/mp.2016.3

7. Fernandes BS, Steiner J, Bernstein HG, Dodd S, Pasco JA, Dean OM, et al. C-reactive protein is increased in schizophrenia but is not altered by antipsychotics: meta-analysis and implications. Mol Psychiatry. (2016) 21:554-64. doi: 10.1038/mp.2015.87

8. Chiappelli J, Notarangelo FM, Pocivavsek A, Thomas MAR, Rowland LM, Schwarcz R, et al. Influence of plasma cytokines on kynurenine and kynurenic acid in schizophrenia. Neuropsychopharmacology. (2018) 43:167580. doi: 10.1038/s41386-018-0038-4

9. Erhardt AS, Schwieler L, Imbeault S, Engberg G. The kynurenine pathway in schizophrenia and bipolar disorder. Neuropharmacology. (2017) 112:297306. doi: 10.1016/j.neuropharm.2016.05.020

10. Nilsson LK, Linderholm KR, Engberg G, Paulson L, Blennow K, Lindström $\mathrm{LH}$, et al. Elevated levels of kynurenic acid in the cerebrospinal fluid of male patients with schizophrenia. Schizophr Res. (2005) 80:31522. doi: 10.1016/j.schres.2005.07.013

11. Linderholm KR, Skogh E, Olsson SK, Dahl ML, Holtze M, Engberg G, et al. Increased levels of kynurenine and kynurenic acid in the CSF of patients with schizophrenia. Schizophr Bull. (2012) 38:426-32. doi: 10.1093/schbul/sbq086

12. Schwieler L, Larsson MK, Skogh E, Kegel ME, Orhan F, Abdelmoaty S, et al. Increased levels of IL-6 in the cerebrospinal fluid of patients with chronic schizophrenia-significance for activation of the kynurenine pathway. J Psychiatry Neurosci. (2015) 40:126-33. doi: 10.1503/jpn.14 0126

13. Kindler J, Lim CK, Weickert CS, Boerrigter D, Galletly C, Liu $\mathrm{D}$, et al. Dysregulation of kynurenine metabolism is related to proinflammatory cytokines, attention, and prefrontal cortex volume in schizophrenia. Mol Psychiatry. (2020) 25:2860-72. doi: 10.1038/s41380-0190401-9

14. Cao B, Chen Y, Ren Z, Pan Z, McIntyre RS, Wang D. Dysregulation of kynurenine pathway and potential dynamic changes of kynurenine in schizophrenia: a systematic review and meta-analysis. Neurosci Biobehav Rev. (2021) 123:203-14. doi: 10.1016/j.neubiorev.2021.01.018

15. American Psychiatric Association. Diagnostic and Statistical Manual of Mental Disorders. 5th ed. Arlington, VA: American Psychiatric Association Publishing Inc. (2013)

16. Kay SR, Fiszbein A, Opler LA. The positive and negative syndrome scale (PANSS) for schizophrenia. Schizophr Bull. (1987) 13:261-76. doi: 10.1093/schbul/13.2.261

17. Inada T. Evaluation and diagnosis of drug-induced extrapyramidal symptoms. In: Yagi G, editor. Commentary on the DIEPSS and Guide to Its Usage. Tokyo: Seiwa Publishers (1996). p. 11-60.

18. Soga T, Heiger DN. Amino acid analysis by capillary electrophoresis electrospray ionization mass spectrometry. Anal Chem. (2000) 72:123641. doi: 10.1021/ac990976y

19. Soga T, Ueno Y, Naraoka H, Ohashi Y, Tomita M, Nishioka T. Simultaneous determination of anionic intermediates for Bacillus subtilis metabolic pathways by capillary electrophoresis electrospray ionization mass spectrometry. Anal Chem. (2002) 74:2233-9. doi: 10.1021/ac02 $0064 n$
20. Soga T, Ohashi Y, Ueno Y, Naraoka H, Tomita M, Nishioka T. Quantitative metabolome analysis using capillary electrophoresis mass spectrometry. $J$ Proteome Res. (2003) 2:488-94. doi: 10.1021/pr034020m

21. Sugimoto M, Wong DT, Hirayama A, Soga T, Tomita M. Capillary electrophoresis mass spectrometry-based saliva metabolomics identified oral, breast and pancreatic cancer-specific profiles. Metabolomics. (2010) 6:7895. doi: 10.1007/s11306-009-0178-y

22. Kanda Y. Investigation of the freely-available easy-to-use software "EZR" (Easy R) for medical statistics. Bone Marrow Transplant. (2013) 48:452-8. advance online publication 3 December 2012; doi: 10.1038/bmt.2012.244

23. Roman M, Irwin MR. Novel neuroimmunologic therapeutics in depression: a clinical perspective on what we know so far. Brain Behav Immun. (2020) 83:7-21. doi: 10.1016/j.bbi.2019.09.016

24. Mohamed BM, Aboul-Fotouh S, Ibrahim EA, Shehata H, Mansour AA, Yassin NA, et al. Effects of pentoxifylline, 7-nitroindazole, and imipramine on tumor necrosis factor- $\alpha$ and indoleamine 2,3-dioxygenase enzyme activity in the hippocampus and frontal cortex of chronic mild-stress-exposed rats. Neuropsychiatr Dis Treat. (2013) 9:697-708. doi: 10.2147/NDT.S41020

25. Jiang X, Xu L, Tang L, Liu F, Chen Z, Zhang J, et al. Role of the Indoleamine2,3-dioxygenase/kynurenine pathway of tryptophan metabolism in behavioral alterations in a hepatic encephalopathy rat model. J Neuroinflammation. (2018) 15:1-6. doi: 10.1186/s12974-017-1037-9

26. Upthegrove R, Manzanares-Teson N, Barnes NM. Cytokine function in medication-naive first episode psychosis: a systematic review and metaanalysis. Schizophr Res. (2014) 155:101-8. doi: 10.1016/j.schres.2014.03.005

27. Miller BJ, Buckley P, Seabolt W, Mellor A, Kirkpatrick B. Meta-analysis of cytokine alterations in schizophrenia: clinical status and antipsychotic effects. Biol Psychiatry. (2011) 70:663-71. doi: 10.1016/j.biopsych.2011.04.013

28. Zhou X, Tian B, Han HB. Serum interleukin-6 in schizophrenia: a system review and meta-analysis. Cytokine. (2021) 141:155441. doi: 10.1016/j.cyto.2021.155441

29. Orlovska-Waast S, Köhler-Forsberg O, Brix SW, Nordentoft M, Kondziella D, Krogh J, et al. Cerebrospinal fluid markers of inflammation and infections in schizophrenia and affective disorders: a systematic review and meta-analysis. Mol Psychiatry. (2019) 24:869-87. doi: 10.1038/s41380-018-0220-4

30. Iaccarino HF, Suckow RF, Xie S, Bucci DJ. The effect of transient increases in kynurenic acid and quinolinic acid levels early in life on behavior in adulthood: implications for schizophrenia. Schizophr Res. (2013) 150:3927. doi: 10.1016/j.schres.2013.09.004

31. Guillemin GJ. Quinolinic acid: neurotoxicity. FEBS J. (2012) 279:1355. doi: 10.1111/j.1742-4658.2012.08493.x

32. Dvojkovic A, Perkovic MN, Sagud M, Erjavec GN, Peles AM, Strac DS, et al. Effect of vortioxetine vs. escitalopram on plasma BDNF and platelet serotonin in depressed patients. Prog Neuropsychopharmacol Biol Psychiatry. (2021) 105:110016. doi: 10.1016/j.pnpbp.2020.110016

33. Sagud M, Perkovic MN, Vuksan-Cusa B, Maravic A, Strac DS, Peles AM, et al. A prospective, longitudinal study of platelet serotonin and plasma brain-derived neurotrophic factor concentrations in major depression: effects of vortioxetine treatment. Psychopharmacol (Berl). (2016) 233:325967. doi: 10.1007/s00213-016-4364-0

34. Stone TW. Neuropharmacology of quinolinic and kynurenic acids. Pharmacol Rev. (1993) 45:309-79.

35. Marx W, McGuinness AJ, Rocks T, Ruusunen A, Cleminson J, Walker AJ, et al. The kynurenine pathway in major depressive disorder, bipolar disorder, and schizophrenia: a meta-analysis of 101 studies. Mol Psychiatry. (2020) 23:1-21. doi: 10.1038/s41380-020-00951-9

36. Luo $\mathrm{Y}, \mathrm{He} \mathrm{H}$, Zhang J, Ou Y, Fan N. Changes in serum TNF$\alpha$, IL-18, and IL-6 concentrations in patients with chronic schizophrenia at admission and at discharge. Compr Psychiatry. (2019) 90:82-7. doi: 10.1016/j.comppsych.2019.01.003

37. Morrens M, De Picker L, Kampen JK, Coppens V. Blood-based kynurenine pathway alterations in schizophrenia spectrum disorders: a meta-analysis. Schizophr Res. (2020) 223:43-52. doi: 10.1016/j.schres.2020.09.007

38. Hestad KA, Engedal K, Whist JE, Farup PG. The relationships among tryptophan, kynurenine, indoleamine 2,3-dioxygenase, depression, and neuropsychological performance. Front Psychol. (2017) 8:1561. doi: 10.3389/fpsyg.2017.01561

39. Maxeiner HG, Marion Schneider E, Kurfiss ST, Brettschneider J, Tumani H, Bechter K. Cerebrospinal fluid and serum cytokine profiling to detect immune 
control of infectious and inflammatory neurological and psychiatric diseases. Cytokine. (2014) 69:62-7. doi: 10.1016/j.cyto.2014.05.008

40. Pillai A, Kale A, Joshi S, Naphade N, Raju MS, Nasrallah H, Mahadik SP. Decreased BDNF levels in CSF of drug-naive first-episode psychotic subjects: correlation with plasma $\mathrm{BDNF}$ and psychopathology. Int $J$ Neuropsychopharmacol. (2010) 13:535-9. doi: 10.1017/S1461145709991015

41. Miller CL. The chemical interaction between adrenochrome, three different classes of antipsychotic drugs and metabolites of the kynurenine pathway. Eur Neuropsychopharmacol. (2015) 25:435-40. doi: 10.1016/j.euroneuro.2014.12.001

Conflict of Interest: The authors declare that the research was conducted in the absence of any commercial or financial relationships that could be construed as a potential conflict of interest.
Publisher's Note: All claims expressed in this article are solely those of the authors and do not necessarily represent those of their affiliated organizations, or those of the publisher, the editors and the reviewers. Any product that may be evaluated in this article, or claim that may be made by its manufacturer, is not guaranteed or endorsed by the publisher.

Copyright (c) 2021 Okamoto, Natsuyama, Igata, Konishi, Tesen, Ikenouchi and Yoshimura. This is an open-access article distributed under the terms of the Creative Commons Attribution License (CC BY). The use, distribution or reproduction in other forums is permitted, provided the original author(s) and the copyright owner(s) are credited and that the original publication in this journal is cited, in accordance with accepted academic practice. No use, distribution or reproduction is permitted which does not comply with these terms. 